\title{
DESIGN EM MOVIMENTO: REPENSANDO O DESIGN ATRAVÉS DO ENCONTRO COM AS CIÊNCIAS SOCIAIS
}

\author{
Cássia Mota \\ Doutoranda em Design, PPD ESDI UERJ \\ cassiamota@gmail.com \\ Marina Sirito \\ Mestranda em Design, PPD ESDI UERJ \\ marina.sirito@gmail.com
}

Resumo: $\mathrm{O}$ artigo busca debater sobre as questões recentes relativas ao desenvolvimento do campo do design através das contribuições das ciências sociais, especialmente de um campo denominado Design Anthropology. Iniciaremos a discussão sobre a abertura que o próprio campo do design fez às interações com outros campos da ciência, além das ciências exatas, entendendo essas interações como movimentos feitos pelo campo para sua reformulação, resignificando o papel do designer, tanto na academia quanto na atividade profissional. Posteriormente, esclareceremos as definições e principais questões debatidas no campo do Design Anthropology. Por fim, exibiremos as principais questões das pesquisas levadas por integrantes do Laboratório de Design e Antropologia (LaDA) da Escola Superior de Desenho Industrial (ESDI) da Universidade Estadual do Rio de Janeiro (UERJ).

Palavras-chave: pesquisa em design, ciências sociais, Design Anthropology.

\begin{abstract}
This article aims to debate some recent questions related to the development of the field of design through the contributions from the social sciences, specailly from a field called Design Anthropology. We'll iniciate the debate with the inflexion that the field of Design made to open up and interact with other scieces, other than exact sciences. Then, we'll clear up some definitions and main questions that are taken within the field of Design Anthropology. Finally, we'll show the developpment of the resarch projetcs that are lead by members of Laboratório de Design e Antropologia (LaDA) from Escola Superior de Desenho Industrial (ESDI) of Universidade Estadual do Rio de Janeiro (UERJ).
\end{abstract}

Keywords: design research, social sciences, Design Anthropology, LaDA. 


\section{INTRODUÇÃO}

Como qualquer campo de conhecimento, o Design está constantemente sendo transformado a partir da prática daqueles que estão profissionalmente engajados na sua atividade. Então, como a própria vida, o design está o tempo todo em movimento. Evoluindo a partir da interação com outros campos da ação humana e os seus modos de produção de conhecimento, o design é atravessado por um processo contínuo de transformação. Contudo, ainda assim, seria possível discernir alguns pontos em meio a este processo que marcaram inflexões significativas para o campo, marcas identificadas e reconhecidas pela comunidade profissional como limites entre os diferentes períodos em meio à história do design.Neste artigo, discutiremos a respeito da contínua revisão do campo do design através de dois movimentos importantes: um de dentro para fora e outro de fora para dentro. O primeiro, que é feito internamente por pensadores e teóricos do próprio campo do design, trata de repensar e debater a atuação através das contribuições que a prática traz - designers pensando design. 0 segundo movimento, de fora para dentro, trata de pensar design a partir das contribuições de outros campos de conhecimento e dos encontros entre design e as diversas ciências. Aqui, em especial, daremos ênfase ao encontro entre design e antropologia.

\section{A INSTITUCIONALIZAÇÃO DO CAMPO DO DESIGN NO BRASIL}

O campo do Design no Brasil se instituiu no início década de 1960, quando começaram a surgir associações profissionais, os escritórios especializados e também cursos de nível universitário, através da implementação de dois modelos diferentes de currículo: a FAU-USP e a ESDI (Anastassakis, 2011), ambos inseridos num paradigma moderno e informados pelas ideias da Bauhaus e Ulm.

A Esdi, cujo modelo foi adotado como parâmetro para a criação de muitos cursos de design no Brasil (Couto, 2008), nos quais se formaram os primeiros profissionais brasileiros, pretendia, em sua formulação, construir-se como a escola de design moderno no país. Segundo Souza Leite (2006), o modelo alemão empregado na Esdi não se preocupava em dialogar com o contexto que estava operando, e essa postura levou a diversos questionamentos em diversos âmbitos ao longo do tempo.

A escolha pelo uso da matriz alemã de cientificidade no ensino do design e sua opção pela universalidade da linguagem formal, impediu um olhar mais atento às circunstâncias precisas do país onde fora instalada, ficando responsável pelo afastamento do "design do mundo real" (Anastassakis, 2011), desconectando a atividade e aqueles que a desempenham da sociedade e cultura na qual se inserem e atuam, e ainda os destituindo da crítica de seu próprio campo de atuação (Souza Leite, 2006).

Entre 1990 e 2000, se dá início a historiografia do design no Brasil, dando ao debate do campo novos limites e significados a partir da articulação de uma série de pesquisas que buscam ampliar a compreensão sobre o campo do design nacional, criando a chance de novos espaços e possibilidades para a prática da profissão. Neste momento, o design brasileiro se volta para uma reflexão sobre sua história, sobre os paradigmas que fundamentaram sua instituição no país e sobre as questões que nortearam o desenvolvimento do campo no Brasil, com o intuito de compreender 
como deverá atuar face aos desafios presentes e futuros, caminhando para uma autoreflexão (Anastassakis, 2011).

Essa auto-reflexão se dá em forma de revisão crítica desencadeada pelo crescimento dos cursos de graduação no país e pelo aumento exponencial de profissionais de design atuando no cenário brasileiro, tanto no âmbito prático quanto teórico, fazendo com que a estruturação do campo seja constantemente questionada.

Nesse processo de revisão, há também uma busca por uma contextualização cultural para o design no Brasil (Anastassakis, 2011), que faz emergir o debate sobre os significados sociais do design e sua adequação ao cenário cultural do Brasil, buscando, com isso, contribuições de outros campos do saber.

\section{O DESIGN E O PARADIGMA DE CIENTIFICIDADE}

Para Binder, professor em codesign na Royal Danish Academy of Fine Arts, inicialmente a grande maioria dos escritos em design, estavam atrelados a um ethos extremamente racionalista, que assumia posicionamento claro em relação a um paradigma de cientificidade que foi particularmente forte por volta dos anos 1950 e 1960, onde a Bauhaus exercia um papel importante na relação entre o design e a industrialização crescente (Binder et al, 2010).

Em relação a esse pensamento puramente racionalista, ancorado na noção de cientificidade vinda das ciências exatas, podemos destacar dois expoentes: Herbert Simon e Buckminster Fuller. Para Frideman (2000:10), em Fuller, o processo de design era visto como uma sequência lógica, com etapas reconhecidas que seguiam um certo fluxo de eventos. Tal objetividade nos aponta para uma compreensão do design como processo lógico, estruturado e passível de repetição, independentemente do contexto e sistema/objeto a ser projetado.

Já Simon, segundo Binder et al (2010:15), muito contribuiu para aproximar o exercício do design a uma análise de sistemas e operações. De acordo com Ken Friedman (2000:11), Herbert Simon caracteriza o design como um processo: o processo a partir do qual empreende-seações que objetivam mudar "situações existentes" em "situações preferidas". Um processo com objetivos e, por sua vez, um processo objetivo.

Tanto em Simon, quanto em Fuller, vemos portanto um certo ethos paradigmático da noção científica de design. Por que paradigmático? Segundo Binder et al (2010:166), todo um zeitgeist da época corroboravam esse viés racionalista de entendimento do mundo, e tal noção era aceita pela grande maioria de pensadores no design quase sem questionamentos, até porque a universidade, naquele momento pós guerra (anos 50-60), era dominada por homens brancos, quase todos com formação nas engenharias, por exemplo.

\section{A INTEGRAÇÃO DO DESIGN COM AS CIÊNCIAS SOCIAIS E HUMANAS}

Contudo, ainda de acordo com Binder et al (2010:166), esse apelo à cientificidade e ao racionalismo no design durou pouco. Mesmo que tenha sido dominante a ideia de um campo do design atrelado à indústria desde a instituição do campo do design, a visão racionalista do mundo foi aos poucos sendo descartada por muitos dos pensadores do campo. A princípio, essa visão foi sendo refutada pelas humanidades e ciências sociais inicialmente por razões políticas. Em grande parte, esse 
confrontamento foi também alavancado pelo aumento da diversidade no meio acadêmico, pela vinda das mulheres, estudantes de classes populares e novos grupos étnicos para a universidade - o que se chocava com a visão universalista antes defendida por apenas um grupo social dominante, que carregava essa "bandeira" do racionalismo, além da mudança de direção da sociedade, agora mais baseada no consumo, e menos na produção industrial.

Nesse sentido, não só o racionalismo foi questionado, mas também o papel das autoridades, das instituições e do sistema produtivo. Parece que, a partir de certo momento, problemas de ordem ecológica, social e humana foram se complexificando a tal ponto que se provaram capciosos e quase irresolúveis apenas pela ótica do racionalismo (Binder et al, 2010: 16).

Segundo o historiador e teórico em cultura visual Clive Dilnot (1989:220), nos anos 1970, o modernismo começou a perder seu apelo, graças a tensões que passaram a entrar em discussão, como, por exemplo, a relação entre design e os produtores, o comércio, o gosto popular. Ainda de acordo com Dilnot, uma vez que o designer passou a ter de considerar, mais que o seu próprio meio, a sociedade como um todo, sua arena de investigação teve de sofrer uma alteração institucional considerável.

Jorge Frascara, pensador argentino da comunicação visual, apresenta nesse contexto a conexão entre o design e as ciências sociais. Para Frascara (2002:234), as relações entre design, antropologia, psicologia e sociologia apontam para um começo de uma abordagem para o design consciente da necessidade de uma aproximação interdisciplinar, que reconfiguraria as estruturas departamentais e compartimentalizadas que, na academia, nos separam das realidades que nos contêm.

Sobre o encontro das ciências sociais e humanas com o design, especialmente com a pesquisa em design, Binder et al (2010:167) situam esse movimento interdisciplinar no pós-cartesianismo. Segundo os autores, as filosofias pós-cartesianas indicam para os designers uma abordagem de mundo com mais sensibilidade, na qual nosso posicionamento se direciona mais para um entendimento das coisas que necessariamente para uma imposição teórica sobre o mundo à nossa volta.

Uma maneira possível de atuação do design no mundo com mais sensibilidade é a abertura do campo a um movimento de ampliação contínuo em sua prática e também em sua conceituação teórica. $O$ design, se visto pela ótica transdicplinar como aponta Bonfim (in Couto, 2014) no que tange a sua teoria, deve estabelecer uma relação entre os conhecimentos sistematizados e as questões da vida real, fazendo com que teoria e prática se ampliem conjuntamente em seus movimentos, retirando do design o pretenso papel de detentor de conhecimentos.

\section{A CONTÍNUA REDEFINIÇÃO DO CAMPO}

Como dissemos anteriormente, dois movimentos são fundamentais para repensar o campo do design: um interno ao campo e outro externo ao campo. De um lado a ampliação do campo acontece pelas intercessões do design com outras disciplinas, quando buscamos conceituar o design ancorados em outras áreas de conhecimento, por outro também se dá quando propomos rever nossos próprios conceitos a partir de nossas próprias atividades práticas e teóricas.

Para Willian Miller, engenheiro, arquiteto e educador americano, o estabelecimento da base de nossa expressão teórica e prática se dá a partir da maneira como conceituamos design, o termo design pode ser usado tanto como 
substantivo quanto como verbo, pode se referir a um objeto ou coisa quando utilizado como substantivo, e a um processo ou sequência de atividades quando empregado como verbo (Miller, 1988).

Friedman (2000:9), assim como Miller, também conceitua o design como um processo. Segundo o autor, o sentido de design como verbo deve preponderar ao sentido como nome, já que o verbo descreve design como um processo de pensamento e planejamento. O uso do termo ora como verbo ora como substantivo que descreve um processo, para Friedman, exemplifica o status ontológico do design como assunto de pesquisa filosófica.

De acordo com Souza Leite (2001), a resignificação do termo design é também a resignificação do campo, pois quando passamos a entender design como verbo ampliamos as possibilidades do campo expandindo ${ }^{1}$ seu raio de ação o multiplicando por outros campos de conhecimento, agregando as possibilidades do design a estes campos que antes eram sobrepostos pelo mesmo.

Para Rafael Cardoso, vivemos num mundo complexo, num sistema interligado de redes. Para Cardoso, é a partir destas redes e do trabalho em equipe que surgem as melhores soluções para questões complexas. Quando nos contrapomos a este fato ou reagimos a ele, estamos minando qualquer possibilidade de mudar as realidades que nos são dadas. Os designers precisam se libertar da ideia de que podem resolver tudo sozinhos (Cardoso, 2012:23), e assim abrirem o campo para encontros com outras áreas de conhecimento, outros profissionais e até mesmo para aqueles aos quais os projetos se destinam.

Outros diversos teóricos também discutem a redefinição do campo através de outros conceitos. Por exemplo Enzio Manzini (2015), designer italiano, apresenta o conceito de design difuso em seu livro Design When Everybody Designs. Segundo o autor, design difuso é exercido por todos enquanto design expert é exercido por aqueles que foram treinados para serem designers. Neste caso redefinir o design passa por redefinir quem está apto a fazer design.

Para nós, a redefinição do campo, além passar por todas as questões levantadas aqui pelos teóricos citados, passa também pelo encontro com outras ciências. Passa por movimentos inter e transdiciplinares, que expandem o campo a partir da sua permeabilidade a outros saberes que nele se integram e dos saberes que Ihe são próprios e que frutificam em outros campos.

\section{SOBRE DESIGN ANTHROPOLOGY}

Um desses encontros interdisciplinares entre o design e as ciências sociais é o campo emergente chamado Design Anthropology. Segundo Zoy Anastassakis (2014), aproximação acadêmica entre design e antropologia já acontece há pelo menos trinta anos. Contudo, o campo que fusiona as duas áreas de maneira horizontal e não hierárquica - Design Anthropology - surgiu recentemente e ainda está se desenvolvendo. Uma rede de pesquisadores é fundamental nessa fusão das áreas: a Research Network for Design Anthropology, uma colaboração internacional entre pesquisadores e instituições. É importante salientar que a rede nasce de um esforço

\footnotetext{
1] Sobre o conceito de campo expandido, ver Kraus, Rosalind (1979). "Sculpture in the expanded field". In October, n. 8 (Spring). Cambridge, Ma: MIT Press. [ed. Brasileira: "A escultura no campo ampliado" (tradução Elizabeth Baez) in Gávea, n. 1. Rio de Janeiro: 1984]
} 
dos pesquisadores, que já buscam esse contato entre design e antropologia nas suas pesquisas e publicações individuais, para criar redes e espaços de conexão entre mais ideias e pessoas.

Dentre os agentes centrais tanto no papel catalisador do encontro de pesquisadores na área quanto na produção bibliográfica sobre o tema, podemos destacar alguns nomes importantes. Joachim Halse, professor da Royal Danish Acadmy of Fine Arts, que exerce grande papel na formação e promoção da rede. Wendy Gunn, professora associada da University of Southern Denmark; Ton Otto, professor na Aarhus University; Rachel Smith, professora assistente também na Aarhus University e Jared Donovan, pesquisador no Queensland University of Technology - juntos, estes pesquisadores editaram duas coletâneas: Design and Antrhropology (em 2012) e Design Anthropology (em 2013).

$\mathrm{Na}$ segunda coletânea dos autores, organizada por Gunn, Otto and Smith (2013:16), podemos encontrar uma definição do campo trânsito disciplinar:

"Praticantes de Design Anthropology seguem situações dinâmicas e relações sociais e estão atentos a como as pessoas percebem, criam e transformam seus ambientes através das suas atividades diárias. (...) As práticas do Design Anthropology acontecem transversalmente em diferentes escalas e tempos $e$ envolvem muitas disciplinas, cada uma trazendo suas próprias maneiras distintas de fazer e saber." 2

De acordo com Zoy Anastassakis (2015:212), pesquisadora também integrante da rede Research Network for Design Anthropology, é possível prever no campo um potencial para gerar impacto tanto na antropologia quanto no design e, além disso, o novo campo mostra-se como um espaço de renovação para ambas áreas que o originaram, tanto para a teoria como para a prática.

No processo de contínua revisão do design, práticas transdisciplinares que criam convergências como as criadas no Design Anthropology impulsionam o movimento do campo, ampliando horizontes não só da prática profissional mas também da interação daqueles que se envolvem com o campo do design em sua própria cultura.

\section{O LaDA: LABORATÓRIO DE DESIGN E ANTROPOLOGIA}

Ancorado nas discussões levantadas no Design Anthropology, mas também nas questões levantadas por outras áreas que convergem interesses iminentes nas ciências sociais e ciências sociais aplicadas, o Laboratório de Design e Antropologia, surgiu em 2013, apoiado pela FAPERJ.

De acordo com Zoy Anastassakis, coordenadora do LaDA, o laboratório "busca ao mesmo tempo investigar as implicações dos fenômenos sociais urbanos para a prática das ciências sociais e investir na experimentação em torno da interface disciplinar que aproxima, mais especificamente, o design e a antropologia."

\footnotetext{
๑Traduzido livremente do inglês: "Practitioners of design anthropology follow dynamic situations and social relations and are concerned with how people perceive, create, and transform their environments through their everyday activities. (...) Design anthropology practices occur across different scales and timelines and involve many disciplines, each bringing their own distinct ways of knowing and doing ."
} 
A partir de nossa própria abordagem sobre Design Anthropology, acreditamos que, através da abertura da nossa participação a outras diferentes disciplinas e atores, expandimos a maneira como nós, pesquisadores em design, entendemos nossa atuação na academia e no campo profissional. Consideramos fundamental a criação de caminhos para facilitar o fazer de futuros colaborativos, sempre atentas à maneira como as pessoas vivem e criam seus mundos diários, e entendendo o que as pessoas desejam e sonham e, olhando para uma compreensão profunda do social, pretendemos com nossa presença no campo, propor intervenções usando as ferramentas do design para gerar alternativas concretas a questões específicas na vida diária, buscando provocar intencionalmente mudanças sociais possíveis.

\section{LaDA: O QUE FAZEMOS}

Em nosso laboratório, investigamos como podemos projetar coisas juntos (Latour, 2008) a partir de uma perspectiva transdisciplinar, onde a antropologia se reúne com o design. Assim, é a partir deste "encontro" que contribuímos para a discussão sobre o projeto e seu processo na contemporaneidade, através de uma interação específica com modos antropológicos de produção de conhecimento. Com esta agenda principal, desde o início do ano em curso, estamos imersos em um processo de auto-análise, discutindo o que significa, de uma perspectiva de pesquisa em Design Anthropology, seguir os movimentos que afetam a prática do design agora.

Hoje, construímos nossas pesquisas individuais interessadas em assuntos diversos, porém fundamentadas em diversas referências comuns, em especial as referências conceituais e bibliográficas vindas do campo do Design Anthropology. Sob a orientação da professora Zoy Anastassakis, somos onze pesquisadoras.

Como grandes temas, poderíamos agrupar os trabalhos em torno de conceitos diferentes como: codesign, a relação entre cultura e patrimônio, as possibilidades da etnografia e da antropologia no design, a questão da participação cidadã, a cidade e as cartografias possíveis do urbano, o papel das instituições, as relações entre imagens e visualidades. Há, evidentemente, um corpo crítico no debate que permeia as pesquisas, que se envolve por diferentes vias com as teorias e histórias do design.

Nesse corpo crítico, também há questões de cunho teórico-metodológico que partilhamos no LaDA. Uma das referências para explorações de método que fundamenta o esforço de pesquisa coletivo é o XLAB, uma iniciativa apoiada pelo Danish Center for Design Research que culminou num livro organizado pelos pesquisadores escandinavos Thomas Binder, Eva Brandt, Mette Agger Eriksen e Johan Redström. O Xlab nos aponta para um tipo de prática exploratória na qual designers conduzem pesquisa através do fazer (Binder et al, 2011: 21).

Como temas de pesquisa individuais, podemos citar diversos interesses que possibilitam a relação entre design e ciências sociais, em especial antropologia e etnografia. Por exemplo, a trajetória social dos resíduos sólidos; as técnicas vernaculares de manufatura de cuias; o patrimônio cultural em torno do Bumba-meuboi em exposição; design e possíveis caminhos no apoio à gestão de doenças crônicas; design colaborativo na concepção de jogos de engajamento social; colaboração, democracia e design.

Alem desses temas, pontuamos ainda outros: reflexão sobre o papel dos designers no espaço público; design participativo e a inovação social no espaço público; a análise do uso design nos projetos de fomento ao artesanato; narrativas 
bordadas pelo viés colaborativo; design como perspectiva de pensar o patrimônio; o material didático na escola indígena na relação design e educação; entre outros temas.

Como um todo, os trabalhos mais se interessam pelos processos de design que dialogam com as coisas (Ingold, 2012), em oposição a um design meramente interessado na geração de produtos ou serviços, através de aportes vindos tanto do design quanto da antropologia.

Como dissemos na introdução deste artigo, não só o trabalho de pesquisa está em movimento mas também o próprio conceito de design se move, se expande e se transforma dentro de cada uma das pesquisas, que buscam intersecções interdisciplinares em diversas instâncias com o Design Anthropology, as ciências humanas e políticas.

O design em movimento assume que um campo científico/profissional pode ser tratado como um processo formativo fluido, em oposição à ideia de campo como quadro disciplinar fechado em si mesmo e, por ser dinâmico, muda à medida em que é aberto para discussão, reflexão e estudo. É transformado também pelas intercessões que faz com o mundo, em todas as possibilidades de engajamento em suas atividades práticas e teóricas, assim como transforma o mundo no contato que faz com o mesmo.

Nos interessa pensar como a pesquisa em design pode acontecer num cenário em que o design se atualiza nesse duplo movimento, interno e externo, de constante resignificação. Como podemos seguir produzindo conhecimento posicionadas na perspectiva de um universo de pesquisa dinâmico, num enquadramento teórico que seja sólido a ponto de conter, mas flexível a ponto de se transformar.

Além disso, o LaDA busca ampliar a interface com projetos que acontecem fora do ambiente acadêmico, levando as discussões cada vez mais para ambientes externos às condições ideais de pesquisa, propondo a experimentação do que é debatido dentro do laboratório no mundo, através do Design Antrhopology e do codesign, por exemplo, usando, muitas vezes, a cidade do Rio de Janeiro como laboratório. Buscamos assim estabelecer novos debates sobre a disciplina respeitando o movimento contínuo do campo e nos tornando parte dos novos lugares que o design vai alcançando em seus movimentos.

\section{REFERÊNCIAS}

ANASTASSAKIS, Zoy. Triunfos e impasses: Lina Bo Bardi, Aloisio Magalhães e ainstitucionalização do design no Brasil. Rio de Janeiro: UFRJ/Museu Nacional/PPGAS, 2011

ANASTASSAKIS, Zoy. O Design brasileiro através do espelho: Lina Bo Bardi, Aloisio magalhães e a questão da contextualização cultural na historiografia do Design no Brasil. Anales del IAA, 43 (1), 81-93. 2013a. Consultado em 22/04/2016 em <http://www.iaa.fadu.uba. ar/ojs/index.php/anales/article/view/106/94>

ANASTASSAKIS, Zoy. Laboratório de Design e Antropologia: preâmbulos teóricos e práticos. Arcos Design. Rio de Janeiro: PPD ESDI - UERJ. Volume 7 Número 1 Junho. pp. 178-193. 2013b. Consultado em 22/04/16 em: <http://www.epublicacoes.uerj.br/index.php/ arcosdesign>

ANASTASSAKIS, Zoy. Design and Anthropology: an interdisciplinary proposition. In: Fórum Proceedings of fourth international forum of design as a process, Barbacena. 
Universidade Estadual de Minas Gerais, 2014. Disponível em:

<http://uerj.academia.edu/ZoyAnastassakis>. Acesso em: 22/04/16.

ANASTASSAKIS, Zoy. Projeções, provocações e especulações entre design e antropologia no centro da cidade do Rio de Janeiro. In: Ferro, Ligia; Raposo, Otavio; Gonçalves, Renata Sá. (Org.). Expressões artísticas: etnografia e criatividade em espaços atlânticos. 1ed. Rio de Janeiro: Mauad X : Faperj, v. , p. 209-228, 2015.

BINDER, T.; KOSHIKEN, I.; REDSTRÖM, J.; WENSVEEN, S.; ZIMMERMAN, J. Design research through practice: From the lab, field, and showroom. Massachusetts: Morgan Kaufman, 2010

BINDER, T.; BRANDT, E.; ERIKSEN, M. A.;REDSTRÖM, J. XLab. Copenhague: The Danish Design School Press, 2011.

BUCHANAN, Richard. Rhetoric, Humanism and Design in Discovering Design, R. Buchanan and V. Margolin, eds. Chicago, IL: The University of Chicago Press, 1995.

CARDOSO, Rafael. Design para um Mundo Complexo. São Paulo: Cosac Naify, 2012.

COUTO, Rita M. Escritos sobre ensino de design no Brasil. Rio de Janeiro: Rio Book's, 2008.

COUTO, R. M. et al. (Org.). Gustavo Amarante Bomfim: uma coletânea. Rio de Janeiro: Rio Book's, 2014.

DILNOT, Clive. The state of Design History. Part I: Mapping the field. In: MARGOLIN, V (ed). Design discourse: history, theory, criticism. Chicago: The University of Chicago Press, 1989.

FRASCARA, Jorge (ed.). Design and the social sciences:making connections. Boca Raton: CRC Press. 2002.

FRIEDMAN, Ken. Creating design knowledge: from research into practice.

Southborough, MA: Southborough University. 2000.

GUNN, W.; OTTO, T.; SMITH, Rachel C. Design anthropology: theory and practice. 1a edição - Inglaterra: Bloomsblury, 2013.

LATOUR, Bruno. A Cautious Prometheus? A Few Steps Toward a Philosophy of Design (With Special Attention to Peter Sloterdijk). Palestra para a "Networks of Design meeting of the Design History Society," Cornwall. 2008 Disponivel em <http://www.brsuno-latour.fr/ sites/default/files/112-DESIGN-CORNWALL-GB.pdf>. Acesso em: 22/04/16.

INGOLD, Tim. Trazendo as coisas de volta à vida: emaranhados criativos num mundo de materiais. Horizontes Antropológicos, Porto Alegre, ano 18, n. 37, p. 24-44.2012.

MANZINI, Enzio. Design, When Everybody Designs. An Introduction to Design For Social Innovation. Londres, Cambridge: The MIT Press, 2015.

MILLER, William. Definition of Design. Texto acessado em 22/04/16. Disponível em: <http://www.wrmdesign.com/Philosophy/Documents/DefinitionDesign.htmAndgt;>.

Research Network for Design Anthropology, Acesso em: 20/04/2016, disponível em $<$ https://kadk.dk/en/research-network-design-anthropology>. 
SOUZA LEITE, João. Uma outra perspectiva para se estudar design. In Designe, s.n. . Rio de Janeiro: IAV/Univercidade, 2001.

SOUZA LEITE, João. De costas para o Brasil: o ensino de um design internacionalista. In: MELO, C. O design gráfico Brasileiro: anos 60. São Paulo: Cosac Naify, 2006. 